\title{
Vaginal delivery in SARS-CoV-2-infected pregnant women in Northern Italy: a retrospective analysis
}

\author{
E Ferrazzi, ${ }^{\text {a,b }}$ L Frigerio, ${ }^{c}$ V Savasi, ${ }^{\text {d,e }}$ P Vergani, ${ }^{\text {, g }}$ F Prefumo, ${ }^{\text {h,i }}$ (D) S Barresi, ${ }^{c}$ S Bianchi, ${ }^{b}$ E Ciriello, ${ }^{c}$ \\ F Facchinetti, ${ }^{j}$ MT Gervasi, ${ }^{k}$ E lurlaro, ${ }^{a}$ A Kustermann, ${ }^{a}$ G Mangili, ${ }^{\prime}$ F Mosca, ${ }^{b, m}$ L Patanè, ${ }^{c}$

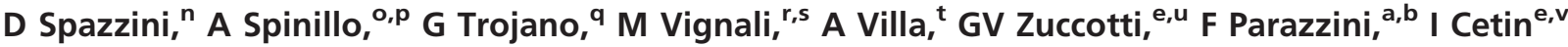

\begin{abstract}
a Department of Woman, Child and Neonate, Fondazione IRCCS Ca' Granda Ospedale Maggiore Policlinico, Milan, Italy ${ }^{\mathrm{b}}$ Department of Clinical Sciences and Community Health, University of Milan, Milan, Italy ${ }^{c}$ Dept of Obstetrics and Gynecology, Papa Giovanni XXIII Hospital, Bergamo, Italy ${ }^{\mathrm{d}}$ Department of Woman, Mother and Neonate, Sacco Hospital-ASST Fatebenefratelli-Sacco, Milan, Italy ${ }^{\mathrm{e}}$ Dept of Biomedical and Clinical Sciences, University of Milan, Milan, Italy ${ }^{\mathrm{f}}$ Department of Maternal Fetal Medicine, Fondazione MBBM, San Gerardo Hospital, Monza, Italy ${ }^{\mathrm{g}}$ University of Milano Bicocca, Monza, Italy ${ }^{\mathrm{h}}$ Department of Obstetrics and Gynecology, Spedali Civili di Brescia, Brescia, Italy ${ }^{i}$ University of Brescia, Brescia, Italy ${ }^{j}$ Obstetrics Unit, Mother Infant Department, AOU of Modena, Modena, Italy ${ }^{\mathrm{k}}$ Obstetrics and Gynecology Unit, Department of Woman's and Child's, Health University Hospital of Padua, Padua, Italy ${ }^{1}$ Department of Neonatology, Papa Giovanni XXIII Hospital, Bergamo, Italy ${ }^{\mathrm{m}}$ Neonatal Intensive Care Unit, Fondazione IRCCS Ca' Granda Ospedale Maggiore, Milano, Italy ${ }^{\mathrm{n}}$ Obstetrics and Gynecology Unit, Azienda Ospedaliera Bolognini, Seriate, Italy ${ }^{\circ}$ Department of Obstetrics and Gynecology, University of Pavia, Pavia, Italy ${ }^{\mathrm{p}}$ IRCCS Foundation Policlinico San Matteo, Pavia, Italy ${ }^{\mathrm{q}}$ Obstetrics and Gynecology Department "Madonna delle Grazie" Hospital, Matera, Italy ${ }^{\mathrm{r}}$ Obstetrics and Gynecology Unit, P.O. Macedonio Melloni-ASST Fatebenefratelli-Sacco, Milan, Italy ${ }^{s}$ Department of Biomedical Sciences for Health, Università degli Studi di Milano, Milan, Italy ${ }^{t}$ Obstetrics and Gynecology Unit, Hospital of Treviglio-Caravaggio, Bergamo, Italy ${ }^{\mathrm{u}}$ Department of Pediatrics, Ospedale dei Bambini V. Buzzi, Milan, Italy ${ }^{\mathrm{v}}$ Department of Woman, Mother and Neonate, Buzzi Children's Hospital-ASST Fatebenefratelli-Sacco Milan, Italy Correspondence: F Parazzini, University of Milan, Via Commenda 12, 20100 Milan, Italy. Email: Fabio.parazzini@unimi.it
\end{abstract}

Accepted 23 April 2020. Published Online 28 May 2020.

This article includes Author Insights, a video abstract available at https://vimeo.com/rcog/authorinsights16278

Objective To report mode of delivery and immediate neonatal outcome in women infected with COVID-19.

Design Retrospective study.

Setting Twelve hospitals in northern Italy.

Participants Pregnant women with COVID-19-confirmed infection who delivered.

Exposure COVID 19 infection in pregnancy.

Methods SARS-CoV-2-infected women who were admitted and delivered from 1 to 20 March 2020 were eligible. Data were collected from the clinical records using a standardised questionnaire on maternal general characteristics, any medical or obstetric co-morbidity, course of pregnancy, clinical signs and symptoms, treatment of COVID 19 infection, mode of delivery, neonatal data and breastfeeding.

Main outcome and measures Data on mode of delivery and neonatal outcome.

Results In all, 42 women with COVID-19 delivered at the participating centres; 24 (57.1\%, 95\% CI 41.0-72.3) delivered vaginally. An elective caesarean section was performed in 18/42
(42.9\%, 95\% CI 27.7-59.0) cases: in eight cases the indication was unrelated to COVID-19 infection. Pneumonia was diagnosed in $19 / 42$ (45.2\%, 95\% CI 29.8-61.3) cases: of these, 7/19 (36.8\%, 95\% CI 16.3-61.6) required oxygen support and 4/19 (21.1\%, 95\% CI 6.1-45.6) were admitted to a critical care unit. Two women with COVID-19 breastfed without a mask because infection was diagnosed in the postpartum period: their newborns tested positive for SARS-Cov-2 infection. In one case, a newborn had a positive test after a vaginal operative delivery.

Conclusions Although postpartum infection cannot be excluded with $100 \%$ certainty, these findings suggest that vaginal delivery is associated with a low risk of intrapartum SARS-Cov-2 transmission to the newborn.

Keywords COVID-19, delivery, transmission.

Tweetable abstract This study suggests that vaginal delivery may be associated with a low risk of intrapartum SARS-Cov-2 transmission to the newborn.

Linked article: This article is commented on by JG Thornton, p. 1122 in this issue. To view this mini commentary visit https://doi.org/10.1111/1471-0528.16308. 
Please cite this paper as: Ferrazzi E, Frigerio L, Savasi V, Vergani P, Prefumo F, Barresi S, Bianchi S, Ciriello E, Facchinetti F, Gervasi MT, Iurlaro E, Kustermann A, Mangili G, Mosca F, Patanè L, Spazzini D, Spinillo A, Trojano G, Vignali M, Villa A, Zuccotti GV, Parazzini F, Cetin I. Vaginal delivery in SARS-CoV-2-infected pregnant women in Northern Italy: a retrospective analysis. BJOG 2020;127:1116-1121.

\section{Introduction}

Most of the information on the effect of COVID-19 infection during pregnancy is based on data relating to other highly pathogenic coronaviruses, i.e. severe acute respiratory syndrome (SARS) and the Middle East respiratory syndrome (MERS). ${ }^{1}$

Recently, Chen et al. ${ }^{2}$ have reported nine cases of deliveries in women with COVID-19 pneumonia. In their study, all nine women had a caesarean section in the third trimester. The neonatal outcomes were favourable and all neonatal throat swabs performed, tested negative for the virus.

Another clinical series of 11 women with COVID 19 infection who had successful deliveries (10 caesarean and 1 vaginal) has been reported: in all the newborns, the 2019nCoV nucleic acid test was negative. ${ }^{3}$

Chen et al. concluded their paper by underlining that 'there is currently no evidence for intrauterine infection caused by vertical transmission in women who develop COVID-19 pneumonia in late pregnancy'. This finding is, however, based on very few reported cases, particularly for vaginally delivered newborns. ${ }^{3,4}$ Accordingly, a recent consensus stated that there is no clear evidence regarding optimal delivery timing, the safety of vaginal delivery or whether caesarean delivery prevents vertical transmission at the time of delivery. The route of delivery and delivery timing should therefore be individualised, based on obstetrical indications and maternal-fetal status. ${ }^{5}$

Due to the recent outbreak of the infection in Italy, particularly in Lombardy, a number of infected women have already delivered. Regione Lombardia, Northern Italy, has established a network of six designated COVID-19 maternity hospitals to offer adequate assistance and epidemiological surveillance to symptomatic, infected pregnant women.

The goal of this study was to report the mode of delivery and immediate neonatal outcome in SARS-CoV-2-infected women observed in the early phase of the epidemic in Lombardy.

\section{Methods}

We performed a retrospective multicentre study of COVID-19-infected women who were admitted and delivered during 1-20 March 2020. Most deliveries of infected women occurred in the designated COVID-19 hubs, but some were delivered in special hospitals because they were in an advanced active phase of labour. The designated Hub-Maternity Hospitals were: Milan-Mangiagalli and Sacco, Bergamo-Pope Giovanni XXIII; Brescia-Civil Hospital; Monza-S. Gerardo Hospital/MBBM Foundation; PaviaSan Matteo. Spokes Maternity Hospitals were: Milan-Melloni and S. Giuseppe; Seriate-Bolognini; Treviglio-Civil Hospital. The Maternity Hospital of Padua and the Maternity Hospital of Modena were not hub hospitals of the Lombardy Region but their cases were also reported for this study.

Criteria for entry to the study were:

- Pregnant women who delivered during the study period with a confirmed diagnosis of COVID-19 infection prior to or within 36 hours after delivery.

The investigators reported all women consecutively observed who met the inclusion criteria.

All centres carefully revised all delivery charts of the study period and all cases who had a confirmed nasopharyngeal swab with an RT-PCR positive test for SARS-CoV-2 infection were included. The clinical triage was performed according to WHO guidelines. ${ }^{6}$ Diagnosis of COVID 19 infection was based on the results of maternal and child nasopharyngeal swab samples according to Italian national procedures. ${ }^{7}$ All women were treated according to the national guidelines for COVID-19 in pregnancy and treatment was then tailored to the individual based on evolution of signs, symptoms, laboratory data and radiological findings. There were no additional obstetric diagnostic procedures or monitoring besides normal clinical practice, apart from a confirmative chest X-ray and 48-hour monitoring of white blood cell count and CRP.

Fetal growth and well-being were assessed at admission and the fetal heart rate was monitored continuously during labour and delivery.

During labour, surgical masks for the labouring woman, her accompanying person and the midwife and/or doctor were worn. More strict personal protective equipment (PPE) was worn during delivery, as the expulsive efforts of bearing down may cause the woman to emit infected droplets.

When the positive infected status of the mother was known at delivery, breast feeding was allowed according to international guidelines ${ }^{8}$ if the mother was asymptomatic or had only minor symptoms. Women were instructed how to wear and dispose of surgical masks, in combination with frequent hand-cleaning with alcohol-based hand rub or soap and water. 
Data were collected from the clinical records using a standardised questionnaire on maternal general characteristics, any medical or obstetric co-morbidity, course of pregnancy, clinical signs and symptoms, treatment of COVID 19 infection, mode of delivery, neonatal data and breastfeeding. In relation to the neonatal outcome, we recorded only whether there was a positive or negative test for COVID-19. Women and newborns were followed up until discharge from hospital or until 25 March, whichever came first.

For the recorded variables, averages, range or proportion and corresponding 95\% CI were computed, as appropriate. Statistically significant differences among groups were tested using the common Chi-square test for heterogeneity.

No core outcome set was used in the research. This research was done without patient involvement. Patients were not invited to comment on the study design and were not consulted to develop patient-relevant outcomes or interpret the results. Patients were not invited to contribute to the writing or editing of this document for readability or accuracy.

The study protocol was approved by the Institutional Review Boards.

This study received no funding.

\section{Results}

A total of 42 women eligible for the study delivered in the participating centres; 32 women delivered at hub hospitals and 10 in the specialised ones.

Diagnosis of COVID-19 infection was known before admission to hospital in 10 cases, in the delivery room in 27 cases and in 5 cases within 36 hours following delivery, while the women were still in hospital.

In all, 24/42 (57.1\%, 95\% CI 41.0-72.3) women delivered vaginally, with three cases undergoing induction of labour for obstetric reasons.

An elective caesarean section was performed in 18/42 (42.9\%, 95\% CI 27.7-59.0) cases: in 8 cases the indication was unrelated to COVID-19 infection but in 10 cases the indications were worsening dyspnoea or other COVID-19related symptoms. In women who attempted vaginal delivery, no emergency caesarean section occurred.

The characteristics, signs, symptoms and treatment of COVID-19 infection of this cohort are presented in Table 1 according to mode of delivery. Mean maternal age was 32.9 (range 21-44). Fever was the most common symptom. Pneumonia was diagnosed in $19 / 42$ (45.2\%, 95\% CI $29.8-$

Table 1. Maternal characteristics and symptoms

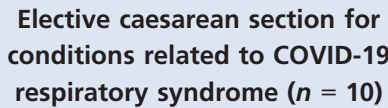

conditions related to COVID-19

respiratory syndrome $(n=10)$

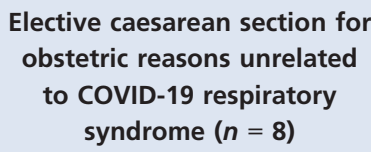

Vaginal delivery* $(n=24)$

\section{Maternal characteristics}

Maternal age, mean (range)

Nulliparous women

Sign and symptoms of SARS-Cov-2 infection

Fever before delivery

Fever only postpartum

Myalgia/malaise

Cough

Dyspnoea

Diarrhoea

Pneumonia

Treatment

Oxygen support (nasal cannula, CPAP)

Admission to critical care unit (yes)

\section{Laboratory findings}

High leucocyte count

$\left(>9.5 \times 10^{9}\right.$ cells/l)**

Lymphopaenia $\left(<10^{9}\right.$ cells/l)**

Elevated C-reactive protein $(>10 \mathrm{mg} / \mathrm{l})^{* *}$

Elevated ALT $(>45 \mathrm{U} / \mathrm{l})$ or AST $(>35 \mathrm{U} / \mathrm{l}))^{* *}$

$30.9(21-40)$
$4(40 \%)$
$7(70 \%)$
-
$5(50 \%)$
$8(80 \%)$
$7(70 \%)$
-
$8(80 \%)$
$4(40 \%)$
$2(20 \%)$
$3(30 \%)$
$3(30 \%)$
$7(70 \%)$
$2(20 \%)$

$\begin{array}{lc}30.5(27-44) & 34.6(29-43) \\ 2(25 \%) & 9(38 \%) \\ 4(40 \%) & 9(38 \%) \\ 1(13 \%) & 5(21 \%) \\ - & 2(8 \%) \\ 2(25 \%) & 8(33 \%) \\ - & 1(4 \%) \\ 1(13 \%) & 1(4 \%) \\ 4(50 \%) & 7(29 \%) \\ 1(13 \%) & 2(8 \%) \\ 1(13 \%) & 1(4 \%) \\ 2(25 \%) & 11(46 \%) \\ 1(13 \%) & 2(8 \%) \\ 4(50 \%) & 6(25 \%) \\ - & 3(13 \%)\end{array}$

Values given as $n$ (\%) unless otherwise indicated.

*In three cases, labour was induced for obstetric indications unrelated to COVID 19 respiratory syndrome.

** Six cases missing. 
Table 2. Pregnancy, delivery and neonatal outcome

\begin{tabular}{|c|c|c|c|}
\hline & $\begin{array}{l}\text { Elective caesarean section for } \\
\text { conditions related to COVID-19 } \\
\text { respiratory syndrome }(n=10)\end{array}$ & $\begin{array}{l}\text { Elective caesarean section } \\
\text { for obstetric reasons unrelated to } \\
\text { COVID-19 respiratory syndrome }(n=8)\end{array}$ & $\begin{array}{l}\text { Vaginal delivery } \\
\qquad(n=24)\end{array}$ \\
\hline \multicolumn{4}{|l|}{ Pregnancy and delivery } \\
\hline Gestational diabetes (yes) & $2(20 \%)$ & - & $4(17 \%)$ \\
\hline \multicolumn{4}{|l|}{ Weeks of gestation at delivery } \\
\hline$>37$ & $5(50 \%)$ & $7(88 \%)$ & $18(78 \%)^{*}$ \\
\hline$>34-37$ & $3(30 \%)$ & - & $4(17 \%)$ \\
\hline$\leq 34$ & $2(20 \%)$ & $1(13 \%)$ & $1(4 \%)$ \\
\hline \multicolumn{4}{|l|}{ Newborn } \\
\hline Birth weight (grams; mean, range)** & $2730(840-4040)$ & $3100(2770-3430)$ & $3226(2450-3740)$ \\
\hline Apgar score $(5 \mathrm{~min})>7$ & $8(80 \%)$ & $8(100 \%)$ & $24(100 \%)$ \\
\hline NICU admission (Yes) & - & $1(13 \%)^{* * *}$ & $2(8 \%)^{* * *}$ \\
\hline Positivity to SARS-Cov-2 (Yes) & 0 & $1(13 \%)$ & $2(8 \%)$ \\
\hline Breastfeeding (Yes) & 0 & $1(12 \%)$ & $10(42 \%)$ \\
\hline
\end{tabular}

61.3) cases. Seven of these 19 (36.8\%, 95\% CI 16.3-61.6) required oxygen support and four of the $19(21.1 \%, 95 \%$ CI 6.1-45.6) were admitted to a critical care unit. Pneumonia was more common in women who delivered by caesarean section due to COVID-19-related infection $\left(\chi^{2}\right.$ $=7.45, P=.024)$.

Table 2 presents the course of pregnancy and the neonatal outcome. Gestational diabetes was reported in 6/42 cases $(14 \%)$ without any significant difference between the three groups. Of the 42 women, 30 delivered at term (71.4\%, 95\% CI 55.4-84.3). Spontaneous preterm birth occurred in five cases and elective caesarean section was performed in six cases.

Two very preterm newborns had a 5-minute Apgar score $<7$; the rest had 5-minute Apgar scores of $\geq 7$.

\section{Newborn outcomes and breastfeeding}

Breastfeeding was allowed In 10 cases, with the women using a surgical mask. Two women had a new diagnosis of COVID-19 infection in the postpartum period and breastfed without a surgical mask; both newborns had a positive test for COVID-19 infection at day 1 and 3, respectively.

In another case after vaginal delivery, the newborn of an infected woman had a positive test. Additional details about this case deserve mention. One newborn from a COVID-19 mother delivered vaginally at term in good condition, was immediately separated from the mother because of a severe maternal postpartum haemorrhage. Within a few hours, the child developed gastrointestinal symptoms. After 3 days, he developed respiratory symptoms and was transferred to the neonatal intensive care unit, where he recovered after 1 day of mechanical ventilation. The first test for SARS-CoV-2 was equivocal a few hours after delivery, but positive 3 days later. The mother did not breastfeed. No associated healthcare providers had a confirmed diagnosis of COVID-19 infection. No other newborn tested positive.

\section{Discussion}

\section{Main findings}

This paper reports the obstetric outcome of a cohort of COVID-19-affected pregnant women and the rate of SARSCoV-2 positivity in newborns according to mode of delivery and breastfeeding status.

The general results show that vaginal delivery occurred in about the $60 \%$ of women. A low risk of intrapartum SARSCov-2 transmission to the newborn cannot be excluded.

The majority of pregnant women affected by the COVID-19 respiratory syndromes suffered mild or moderate symptoms. Fever, cough and mild dyspnoea were the most common symptoms (80\%), but pneumonia was diagnosed in about the $40 \%$ of women.

\section{Strengths and limitations}

Among the strengths of this analysis, we included in our study all consecutive positive women delivered in or admitted to the postpartum COVID-19 ward, in all maternity units of the COVID-network in Lombardy and Units of Padua and Modena, so as to be sure to include all symptomatic cases who tested positive on the nasopharyngeal 
sampling. The reported cases represent approximately $0.6 \%$ of the total deliveries occurring in the same area during the 20 days of the study.

Among the limitations we should underline that due to the limited follow up, the non-immediate maternal and newborn outcome was not considered.

\section{Interpretation}

In our study, the maternal conditions were generally mild to moderate. Radiologically confirmed pneumonia was diagnosed in $42 \%$ of cases and four of these 19 cases required admission to a critical care unit. As suggested by others, ${ }^{9}$ the findings of our cohort support the hypothesis that COVID-19 respiratory syndrome may be less severe for maternal prognosis than were SARS and MERS.

Lymphopaenia and high CRP values were part of the clinical scenario that induced clinicians to deliver patients by caesarean section.

Of note, two cases were delivered $<34$ weeks of gestation because of worsening respiratory function. Five women delivered spontaneously before term (one before 34 weeks of gestation). These observations are compatible with no increase in the risk of preterm birth, consistent with the findings of a previously published series reporting no cases of preterm birth before 33 weeks of gestation. ${ }^{4}$

Vertical and intrapartum transmission are among the most serious complications of viral diseases during pregnancy. In the previously quoted published series of a total of 30 women, ${ }^{2,3}$ delivery in all but one case was by caesarean section. No newborn infection was reported. Vertical transmission does not seem to occur after infection with other pathogenic coronaviruses such as SARS-CoV and MERS-CoV- infection, although it has been suggested that coronaviruses may cause early pregnancy loss. ${ }^{10,11}$

Previous data on virus transmission are based substantially on women delivered by caesarean section. Vertical transmission of viral infection generally occurs during intrauterine life by transfer across the placenta, or during delivery by ingestion or aspiration of cervicovaginal secretions, and in the postpartum period via breastfeeding ${ }^{12 .}$ The risk of ingestion or aspiration of cervical secretion or with contact with perineal-infected tissue is of course higher in the case of vaginal delivery. Among the 24 women who delivered vaginally, one newborn was infected, probably due to postpartum contamination (see below). In a second case after vaginal delivery, a potential intrapartum infection might have occurred but it was not possible to exclude infection immediately postpartum.

It should be emphasised that we can only consider the risk of transmission among women who were infected during the third trimester or at term and the risk of intrapartum transmission, because all infections in Northern Italy are recent and the women infected in early pregnancy are still pregnant.
We report five women who were diagnosed to be COVID-19-positive because of fever in the postpartum period. In two cases in which skin-to-skin contact after birth and breastfeeding was allowed without a mask because infection was not known, the COVID-19 test of the newborn was positive at day 1 and 3 after birth. Although no viral load has been detected in breast milk by Chan et al., ${ }^{2}$ close maternal contact may represent a potential route of transmission. In these two cases, because viral testing was not carried out immediately after birth, vertical transmission cannot be excluded.

To our knowledge, two other cases of SARS-CoV-2-infected newborns have been reported in which the diagnosis was made respectively 36 hours following delivery and at 17 days of life. In both cases, a postpartum neonatal infection acquired through an infected contact was suggested. ${ }^{4}$ In both these cases, because viral testing was not performed immediately after birth, the route of transmission cannot be definitely established.

Elevated IGM antibodies against coronavirus have been also reported by Dong et al. ${ }^{13}$ in a case after caesarean section. Three additional newborns with elevated IGM antibodies to SARS-COv-2 virus, but with a a negative RTPCR throat swab test, have been also reported. ${ }^{14}$ Caution in interpreting these findings has been suggested, including the possibility that IGM positivity could represent a laboratory artefact. ${ }^{15}$

\section{Conclusion}

The frequency of COVID-19 symptoms and positive laboratory and radiological findings observed in this cohort are in line with this syndrome generally being mild or moderate in pregnancy and many infected pregnant women very likely being totally asymptomatic or developing symptoms only after delivery. Vaginal delivery is appropriate in mild cases and caesarean section should be reserved for women with severe respiratory problems, where delivering the baby will allow improved ventilation. Although postpartum infection cannot be excluded, our study also suggests that vaginal delivery may be associated with a low risk of intrapartum SARS-Cov-2 transmission to the newborn. Further data on this finding is needed.

The observed occurrence of COVID-19 symptoms only after delivery suggests that, in areas characterised by a high prevalence of infection, safe procedures for midwives and doctors are to be recommended in any labour as well as adopting mask and safe procedures in all breastfeeding women.

\section{Disclosure of interests}

None declared. Completed disclosure of interests forms are available to view online as supporting information. 


\section{Contribution to authorship}

FP, EF, IC and LF each led aspects of the contact investigation and provided overall leadership and guidance in the investigation. FM, GVZ and GM were the paediatricians in charge of treatment of the newborn babies in the main collaborating centres. LF, VS, PV, SB, FF, MTG, EI, AK, BM, LP, FeP, DS, AS, GT, PV, MV and AV completed the investigation of cases and collected epidemiological data, and provided clinical care to the patients and assisted with clinical descriptions. FP and EF drafted the manuscript. IC revised the manuscript. All authors reviewed and approved the final manuscript.

\section{Details of ethics approval}

The study was approved by the Institutional Review Boards (15408/2020 IRB Milan Area 1).

\section{Funding}

No funding.

\section{Acknowledgements}

For their partnership and dedication, we wish to thank the obstetricians, the midwives and the health providers of the participating centres that in these dramatic days have assisted the women during their deliveries.

\section{Data sharing}

With the permission of the corresponding authors, we provide data without names and identifiers. The corresponding authors have the right to decide whether to share the data based on the research objectives and plan provided. Fabio Parazzini affirms that the manuscript is an honest, accurate and transparent account of the study being reported; that no important aspects of the study have been omitted; and that any discrepancies from the study as planned (and, if relevant, registered) have been explained.

\section{Supporting Information}

Additional supporting information may be found online in the Supporting Information section at the end of the article.

Video S1. Author Insights.

\section{References}

1 Rasmussen SA, Smulian JC, Lednicky JA, Wen TS, Jamieson DJ. Coronavirus Disease 2019 (COVID-19) and pregnancy: what obstetricians need to know. Am J Obstet Gynecol 2020;222:415-26.

2 Chen H, Guo J, Wang C, Luo F, Yu X, Zhang W, et al. Clinical characteristics and intrauterine vertical transmission potential of COVID-19 infection in nine pregnant women: a retrospective review of medical records. Lancet 2020;395:809-15.

3 Liu D, Li L, Wu X, Zheng D, Wang J, Yang L, et al. Pregnancy and perinatal outcomes of women with coronavirus disease (COVID-19) pneumonia: a preliminary analysis. AJR Am J Roentgenol 2020. https://doi.org/10.2214/AJR.20.23072

4 Schwartz DA. An analysis of 38 pregnant women with COVID-19, their newborn infants, and maternal-fetal transmission of SARS-CoV2: maternal coronavirus infections and pregnancy outcomes. Arch Pathol Lab Med 2020. https://doi.org/10.5858/arpa.2020-0901-SA

5 Chen D, Yang H, Cao Y, Cheng W, Duan T. ,Fan C et al. Expert consensus for managing pregnant women and neonates born to mothers with suspected or confirmed novel coronavirus (COVID-19) infection. Int J Gynaecol Obstet. 2020. https://doi.org/10.1002/ijgo.13146

6 World Health Organization. Clinical management of severe acute respiratory infection when novel coronavirus ( $\mathrm{nCoV}$ ) infection is suspected: interim guidance. 2020. https://wwwwhoint/docs/defaultsource/coronaviruse/clinical-management-of-novel-covpdf?sfvrsn=bc 7da517_2. Published on 12 January 2020.

7 http://www.trovanorme.salute.gov.it/norme/renderNormsanPdf? anno $=2020 \&$ codLeg $=73669$ \&parte $=1 \% 20 \&$ serie $=$ null

$8 \mathrm{WHO}$. Home care for patients with suspected novel coronavirus ( $\mathrm{nCoV}$ ) infection presenting with mild symptoms and management of contacts. Retrieved 11 March 2020.

9 Mullins E, Evans D, Viner RM, O'Brien P, Morris E. Coronavirus in pregnancy and delivery: rapid review. Ultrasound Obstet Gynecol 2020. https://doi.org/10.1002/uog.22014

10 Schwartz DA.COVID-19, SARS-CoV-2 and pregnancy: Does the past predict the present? ContagionLive. 28 February 2020. Available from: sarscov2-and-pregnancy-does-the-past-predict-the-present (accessed 29 March 2020).

11 Maxwell C, McGeer A, Young Tai KF, , Sermer M. No. 225Management guidelines for obstetric patients and neonates born to mothers with suspected or probable severe acute respiratory syndrome (SARS). J Obstet Gynaecol Can 39:e130-e137.

12 Silasi M, Cardenas I, Racicot K, Kwon JY, Aldo P, Mor G. Viral infections during pregnancy. Am J Reprod Immunol 2015;73:199-213.

13 Dong L, Tian J, He S, Zhu C, Wang J, Liu C, Yang J. Possible vertical transmission of SARS-CoV-2 from an infected mother to her newborn. JAMA. Published online 26 March 2020. https://doi.org/ 10.1001/jama.2020.4621

14 Zeng H, Xu C, Fan J, Tang Y, Deng Q, Zhang W, Long X. Antibodies in infants born to mothers with COVID-19 pneumonia. JAMA 2020;323:1848-9.

15 Kimberlin DW, Stagno S. Can SARS-CoV-2 infection be acquired in utero? More definitive evidence is needed. JAMA 2020. https://doi. org/10.1001/jama.2020.4868 